\title{
Oculomotor Nerve
}

National Cancer Institute

\section{Source}

National Cancer Institute. Oculomotor Nerve. NCI Thesaurus. Code C12758.

The third cranial nerve that originates originating at the oculomotor nerve nucleus at the level of the superior colliculus in the midbrain, provides motor innervation to the levator palpebrae superioris, the superior, inferior, and medial rectus muscles, and the inferior oblique muscles, and provides parasympathetic innervation to the ciliary gang lion. 